\title{
Inequalities for the Minimum Eigenvalue of Doubly Strictly Diagonally Dominant $M$-Matrices
}

\author{
Ming $\mathrm{Xu},{ }^{1,2}$ Suhua $\mathrm{Li}^{2}$ and Chaoqian $\mathrm{Li}^{2}$ \\ ${ }^{1}$ School of Mathematical Sciences, Kaili University, Kaili 556011, China \\ ${ }^{2}$ School of Mathematics and Statistics, Yunnan University, Kunming 650091, China \\ Correspondence should be addressed to Chaoqian Li; lichaoqian05@163.com
}

Received 28 June 2014; Accepted 31 July 2014; Published 17 August 2014

Academic Editor: Shi-Liang Wu

Copyright (c) 2014 Ming Xu et al. This is an open access article distributed under the Creative Commons Attribution License, which permits unrestricted use, distribution, and reproduction in any medium, provided the original work is properly cited.

Let $A$ be a doubly strictly diagonally dominant $M$-matrix. Inequalities on upper and lower bounds for the entries of the inverse of $A$ are given. And some new inequalities on the lower bound for the minimal eigenvalue of $A$ and the corresponding eigenvector are presented to establish an upper bound for the $\mathscr{L}_{1}$-norm of the solution $x(t)$ for the linear differential system $d x / d t=-A x(t)$, $x(0)=x^{0}>0$.

\section{Introduction}

For a positive integer $n, N$ denotes the set $\{1,2, \ldots, n\}$. For $A=\left[a_{i j}\right] \in R^{n \times n}$, we write $A \geq 0(A>0)$ if all $a_{i j} \geq 0\left(a_{i j}>0\right)$, $i, j \in N$. If $A \geq 0(A>0)$, we say $A$ is nonnegative (positive, resp.). Let $Z_{n}$ denote the class of all $n \times n$ real matrices all of whose off-diagonal entries are nonpositive. A matrix $A$ is called an $M$-matrix [1] if $A \in Z_{n}$ and the inverse of $A$, denoted by $A^{-1}$, is nonnegative.

Let $A$ be an $M$-matrix. Then there exist a positive eigenvalue of $A, \tau(A)=\rho\left(A^{-1}\right)^{-1}$, and a corresponding eigenvector $x=\left[x_{1}, x_{2}, \ldots, x_{n}\right]^{T} \geq 0$, where $\rho\left(A^{-1}\right)$ is the Perron eigenvalue of the nonnegative matrix $A^{-1}, \tau(A)=$ $\min \{|\lambda|: \lambda \in \sigma(A)\}$, and $\sigma(A)$ denotes the spectrum of $A$. $\tau(A)$ is called the minimum eigenvalue of $A[2,3]$. If, in addition, $A$ is irreducible, then $A^{-1}>0$ and $\tau(A)$ is simple and $x>0$, which is unique if we assume that the $\mathscr{L}_{1}$-norm of $x$ equals 1; that is, $\|x\|_{1}=\sum_{i=1}^{n}\left|x_{i}\right|=1$ [3]. If $D$ is the diagonal matrix of an $M$-matrix $A$ and $C=D-A$, then the spectral radius of the Jacobi iterative matrix $J_{A}=D^{-1} C$ of $A$ is denoted by $\rho\left(J_{A}\right)$. For a set $\Omega$, we denote by $|\Omega|$ the cardinality of $\Omega$. Note that $\Omega=\emptyset$ if and only if $|\Omega|=0$.
For convenience, we employ the following notations throughout. Let $A=\left[a_{i j}\right] \in R^{n \times n}$ be nonsingular with $a_{i i} \neq 0$, for all $i \in N$, and $A^{-1}=\left[\beta_{i j}\right]$. We denote, for any $i, j \in N$,

$$
\begin{gathered}
h_{i}=\frac{1}{\left|a_{i i}\right|} \sum_{j \neq i}^{n}\left|a_{i j}\right|, \quad s_{i}=a_{i i}+\sum_{j \neq i}^{n} a_{i j} h_{j}, \\
r_{i}=\frac{1}{\left|a_{i i}\right|} \sum_{j=i+1}^{n}\left|a_{i j}\right|, \quad l_{i}=\frac{1}{\left|a_{i i}\right|} \sum_{j=i+1}^{n}\left|a_{j i}\right|, \\
R_{i}(A)=\sum_{j=1}^{n} a_{i j}, \quad R(A)=\max _{i \in N} R_{i}(A), \\
M=\max _{i \in N}\left\{\sum_{j=1}^{n} \beta_{i j}\right\}, \quad m=\min _{i \in N}\left\{\sum_{j=1}^{n} \beta_{i j}\right\}, \\
\Delta^{-}=\left\{i \in N: h_{i}>1\right\}, \quad \Delta^{0}=\left\{i \in N: h_{i}=1\right\}, \\
\Delta^{+}=\left\{i \in N: h_{i}<1\right\} .
\end{gathered}
$$


Definition 1 (see [4]). A matrix $A=\left[a_{i j}\right] \in C^{n \times n}$ is called

(i) (strictly) diagonally dominant, if $h_{i} \leq 1\left(h_{i}<1\right.$, resp.) for all $i \in N$, and $A$ is called doubly (strictly) diagonally dominant if $h_{i} h_{j} \leq 1\left(h_{i} h_{j}<1\right.$, resp.) for all $i, j \in N, i \neq j$;

(ii) weakly chained diagonally dominant, if $h_{i} \leq 1, J(A)=$ $\left\{i \in N: h_{i}<1\right\} \neq \emptyset$ and for all $i \in N / J(A)$, there exist indices $i_{1}, i_{2}, \ldots, i_{k}$ in $N$ with $a_{i_{r} i_{r+1}} \neq 0,0 \leq r \leq k-1$, where $i_{0}=i$ and $i_{k} \in J(A)$.

Remark. (i) It is well known that a doubly strictly diagonally dominant matrix $A$ is nonsingular and that $\left|\Delta^{-} \cup \Delta^{0}\right| \leq 1$ [5]. If $\left|\Delta^{-} \cup \Delta^{0}\right|=1$, we denote by $i_{0}$ the unique element throughout; that is, $\Delta^{-} \cup \Delta^{0}=\left\{i_{0}\right\}$. Meanwhile, if $A$ is doubly strictly diagonally dominant and $\Delta^{-} \cup \Delta^{0}=\emptyset$, then $A$ is strictly diagonally dominant.

(ii) It is clear that a strictly diagonally dominant matrix is doubly strictly diagonally dominant and also weakly chained diagonally dominant. Also clearly, for a doubly strictly diagonally dominant matrix $A$, if $\Delta^{-}=\emptyset$, then $A$ is weakly chained diagonally dominant; otherwise, $A$ is not weakly chained diagonally dominant.

Estimating the bounds of the minimum eigenvalue $\tau(A)$ of an $M$-matrix $A$ and its corresponding eigenvector is an interesting subject in matrix theory and has important applications in many practical problems; see [4, 6-8]. In particular, these bounds are used to estimate upper bounds of the $\mathscr{L}_{1}$-norm of the solution $x(t)$ for the following system of ordinary differential equations:

$$
\frac{d x}{d t}=-A x(t), \quad x(0)=x^{0}>0,
$$

where $x(t), x^{0} \in R^{n}$, and $A \in R^{n \times n}$ is a constant $M$-matrix. And it is proved in [6] that

$$
\|x(t)\|_{1} \leq Q e^{-\tau(A) t}\left\|x^{0}\right\|_{1},
$$

where $Q=\max _{i, j \in N}\left(z_{i} / z_{j}\right)$ and $z=\left[z_{1}, z_{2}, \ldots, z_{n}\right]^{T}$ is the positive eigenvector of $A^{T}$ corresponding to $\tau(A)$. When the order $n$ of $A$ is large, it is difficult to compute $\tau(A)$ and $z$. Hence it is necessary to estimate the bounds of $\tau(A)$ and $z$.

In [4], Shivakumar et al. obtained the following bounds of $\tau(A)$ when $A$ is a weakly chained diagonally dominant $M$ matrix.

Theorem 2 (see [4, Theorem 4.1]). Let $A=\left[a_{i j}\right] \in R^{n \times n}$ be a weakly chained diagonally dominant $M$-matrix and $A^{-1}=$ $\left[\beta_{i j}\right]$. Then

$$
\begin{gathered}
r(A) \leq \tau(A) \leq R(A), \quad \tau(A) \leq \min _{i \in N} a_{i i}, \\
\frac{1}{M} \leq \tau(A) \leq \frac{1}{m} .
\end{gathered}
$$

Recently, Tian and Huang [9] provided lower bounds of $\tau(A)$ by using the spectral radius of the Jacobi iterative matrix $J_{A}$ for a general $M$-matrix $A$.
Theorem 3 (see [9, Theorem 3.1]). Let $A=\left[a_{i j}\right] \in R^{n \times n}$ be an $M$-matrix and $A^{-1}=\left[\beta_{i j}\right]$. Then

$$
\tau(A) \geq \frac{1}{1+(n-1) \rho\left(J_{A}\right)} \frac{1}{\max _{i \in N}\left\{\beta_{i i}\right\}} .
$$

Also in [9], a lower bound of $\tau(A)$, which depends only on the entries of $A$, has been presented when $A$ is a strictly diagonally dominant $M$-matrix.

Theorem 4 (see $\left[9\right.$, Corollary 3.4]). Let $A=\left[a_{i j}\right] \in R^{n \times n}$ be $a$ strictly diagonally dominant $M$-matrix. Then

$$
\tau(A) \geq \frac{1}{1+(n-1) \max _{i \in N}\left\{h_{i}\right\}} \min _{i \in N}\left\{s_{i}\right\} .
$$

As shown in [9], it is possible that $r(A)$ equals zero or that $1 / M$ is very small, and moreover, whenever $A$ is not weakly chained diagonally dominant, Theorems 2 and 4 cannot be used to estimate the bounds of $\tau(A)$ effectively. On the other hand, it is difficult to estimate $\tau(A)$ by using Theorem 3 because of the difficulty of computing the diagonal elements of $A^{-1}$ and $\rho\left(J_{A}\right)$ when $n$ is very large.

In this paper, we continue to research the problems mentioned previously. For a doubly strictly diagonally dominant $M$-matrix $A$, we in Section 3 give some inequalities on the bounds of the entries of $A^{-1}$. And in Section 4, some inequalities on bounds of $\tau(A)$ and the corresponding eigenvector are established. Lastly, an example, in which we estimate the $\mathscr{L}_{1}$-norm of the solution for the system (2) when $A$ is a doubly strictly diagonally dominant $M$-matrix, is given in Section 5.

\section{Preliminaries}

In this section, we give a lemma which involves some results for a doubly strictly diagonally dominant $M$-matrix. First, some notations are listed: for a doubly strictly diagonally dominant matrix $A=\left[a_{i j}\right] \in R^{n \times n}$ and $i, j \in N$,

$\widehat{h}_{i}$

$$
= \begin{cases}h_{i}, & \text { if }\left(\Delta^{-} \bigcup \Delta^{0}\right)=\emptyset, \\ h_{i}, & \text { if } i \in\left(\Delta^{-} \bigcup \Delta^{0}\right)=\left\{i_{0}\right\}, \\ \frac{1}{\left|a_{i i}\right|}\left(\left|a_{i i_{0}}\right| h_{i_{0}}+\sum_{j \neq i, i_{0}}\left|a_{i j}\right|\right), & \text { if } i \notin\left(\Delta^{-} \bigcup \Delta^{0}\right)=\left\{i_{0}\right\},\end{cases}
$$

$$
\widehat{s}_{i}= \begin{cases}s_{i}, & \text { if }\left(\Delta^{-} \bigcup \Delta^{0}\right)=\emptyset, \\ a_{i i}+\frac{1}{\widehat{h}_{i}} \sum_{j \neq i} a_{i j} \widehat{h}_{j}, & \text { if } i \in\left(\Delta^{-} \bigcup \Delta^{0}\right)=\left\{i_{0}\right\}, \\ a_{i i}+\sum_{j \neq i} a_{i j} \widehat{h}_{j}, & \text { if } i \notin\left(\Delta^{-} \bigcup \Delta^{0}\right)=\left\{i_{0}\right\},\end{cases}
$$




$$
\widehat{r}_{i}=\left\{\begin{array}{l}
r_{i}, \quad \text { if }\left(\Delta^{-} \bigcup \Delta^{0}\right)=\emptyset, \\
\frac{1}{\omega\left|a_{i i}\right|} \sum_{j=i+1}\left|a_{i j}\right|, \\
\quad \text { if }\left(\Delta^{-} \bigcup \Delta^{0}\right)=\left\{i_{0}\right\}, i=i_{0}, \\
\frac{1}{\left|a_{i i}\right|}\left(\sum_{j=i+1, j \neq i_{0}}\left|a_{i j}\right|+\left|a_{i i_{0}}\right| h_{i_{0}}\right), \\
\quad \text { if }\left(\Delta^{-} \bigcup \Delta^{0}\right)=\left\{i_{0}\right\}, i<i_{0}, \\
r_{i}, \quad \text { if }\left(\Delta^{-} \bigcup \Delta^{0}\right)=\left\{i_{0}\right\}, i>i_{0},
\end{array}\right.
$$

where

$$
\omega= \begin{cases}1, & \text { if }\left(\Delta^{-} \bigcup \Delta^{0}\right)=\emptyset, \\ \min _{i \neq i_{0}} \frac{1}{h_{i}}, & \text { if }\left(\Delta^{-} \bigcup \Delta^{0}\right)=\left\{i_{0}\right\} .\end{cases}
$$

Note here that let $1 / h_{i}=+\infty$ if $h_{i}=0\left(i \neq i_{0}\right)$.

Lemma 5. Let $A=\left[a_{i j}\right] \in R^{n \times n}$ be a doubly strictly diagonally dominant $M$-matrix and $\left(\Delta^{-} \cup \Delta^{0}\right)=\left\{i_{0}\right\}$. And, for any $\varepsilon \in$ $\left(h_{i_{0}}, \min _{i \neq i_{0}}\left(1 / h_{i}\right)\right)$, let $X=\operatorname{diag}\left(x_{1}, x_{2}, \ldots, x_{n}\right)$, where $x_{i_{0}}=\varepsilon$ and $x_{i}=1, i \neq i_{0}$. Then $A X$ is a strictly diagonally dominant $M$-matrix. Furthermore, $\widehat{h}_{i_{0}} \geq 1, \widehat{h}_{i}<1$ for $i \neq i_{0}$ and $\widehat{s}_{i}>0$ for any $i \in N$.

Proof. Since $A$ is a doubly strictly diagonally dominant $M$ matrix and $\left(\Delta^{-} \cup \Delta^{0}\right)=\left\{i_{0}\right\}$, we have

$$
1 \leq h_{i_{0}}<\min _{i \neq i_{0}} \frac{1}{h_{i}}
$$

hence, from $\varepsilon \in\left(h_{i_{0}}, \min _{i \neq i_{0}}\left(1 / h_{i}\right)\right)$,

$$
\frac{1}{a_{i_{0} i_{0}} \varepsilon} \sum_{j \neq i_{0}}\left|a_{i_{0} j}\right|<1 .
$$

And, for any $i \neq i_{0}$, if $\sum_{j \neq i}\left|a_{i j}\right| \neq 0$,

$$
1<\frac{a_{i i}}{\varepsilon \sum_{j \neq i}\left|a_{i j}\right|} \leq \frac{a_{i i}}{\left|a_{i i_{0}}\right| \varepsilon+\sum_{j \neq i, i_{0}}\left|a_{i j}\right|},
$$

and if $\sum_{j \neq i}\left|a_{i j}\right|=0$, inequality (11) is obvious.

From inequality (11), we have

$$
\frac{\left|a_{i i_{0}}\right| \varepsilon+\sum_{j \neq i, i_{0}}\left|a_{i j}\right|}{a_{i i}}<1, \quad i \neq i_{0} .
$$

Let $A X=\left[\bar{a}_{i j}\right]$. Then

$$
\bar{a}_{i j}= \begin{cases}a_{i j} \varepsilon, & j=i_{0}, i \in N, \\ a_{i j}, & j \neq i_{0}, i \in N .\end{cases}
$$

From inequality (10), we have

$$
\frac{1}{\bar{a}_{i_{0} i_{0}}} \sum_{j \neq i_{0}}\left|\bar{a}_{i_{0} j}\right|=\frac{1}{a_{i_{0} i_{0}} \varepsilon} \sum_{j \neq i_{0}}\left|a_{i_{0} j}\right|<1 .
$$

And, for any $i \neq i_{0}$, from inequality (12), we have

$$
\frac{1}{\bar{a}_{i i}} \sum_{j \neq i}\left|\bar{a}_{i j}\right|=\frac{\left|a_{i i_{0}}\right| \varepsilon+\sum_{j \neq i, i_{0}}\left|a_{i j}\right|}{a_{i i}}<1 .
$$

From inequality (14) and inequality (15), $A X$ is strictly diagonally dominant. Moreover, it is clear that $A X \in Z_{n}$ and $(A X)^{-1}=X^{-1} A^{-1} \geq 0$, which implies that $A X$ is an $M$ matrix.

Furthermore, from the definition of $\widehat{h}_{i}$, we have that

$$
1 \leq h_{i_{0}}=\widehat{h}_{i_{0}}
$$

and for any $i \neq i_{0}$,

$$
\widehat{h}_{i}=\frac{\left|a_{i i_{0}}\right| h_{i_{0}}+\sum_{j \neq i, i_{0}}\left|a_{i j}\right|}{a_{i i}} \leq \frac{\left|a_{i i_{0}}\right| \varepsilon+\sum_{j \neq i, i_{0}}\left|a_{i j}\right|}{a_{i i}}<1 .
$$

We now prove $\widehat{s}_{i}>0$ for any $i \in N$. Since $A$ is doubly strictly diagonally dominant, we get that there is $k \in N, k \neq i_{0}$, such that $a_{i_{0} k} \neq 0$ (otherwise, a contradiction to the definition of doubly strictly diagonally dominant matrices). Hence

$$
0=a_{i_{0} i_{0}} h_{i_{0}}-\sum_{j \neq i_{0}}\left|a_{i_{0} j}\right|<a_{i_{0} i_{0}} h_{i_{0}}-\sum_{j \neq i_{0}}\left|a_{i_{0} j}\right| \widehat{h}_{j},
$$

and equivalently,

$$
\widehat{s}_{i_{0}}=a_{i_{0} i_{0}}-\frac{1}{\widehat{h}_{i_{0}}} \sum_{j \neq i_{0}}\left|a_{i_{0} j}\right| \widehat{h}_{j}>0 .
$$

And for any $i \neq i_{0}$,

$$
\begin{aligned}
\widehat{s}_{i}=a_{i i}-\sum_{j \neq i_{0}}\left|a_{i j}\right| \widehat{h}_{j} & =a_{i i}-\left(\left|a_{i i_{0}}\right| \widehat{h}_{i_{0}}+\sum_{j \neq i, i_{0}}\left|a_{i j}\right| \widehat{h}_{j}\right) \\
& \geq a_{i i}-\left(\left|a_{i i_{0}}\right| h_{i_{0}}+\sum_{j \neq i, i_{0}}\left|a_{i j}\right|\right) \\
& >0 \text { (by Inequality (17)). }
\end{aligned}
$$

Hence, from inequality (19), inequality (20), and the fact that $A$ is an $M$-matrix, we have that, for any $i \in N$,

$$
\widehat{s}_{i}>0 .
$$

The proof is completed.

Lemma 6 (see [10, Page 719]). Let $A=\left[a_{i j}\right]$ be an $n \times n$ complex matrix and let $x_{1}, x_{2}, \ldots, x_{n}$ be positive real numbers. Then all the eigenvalues of $A$ lie in the

$$
\bigcup_{i}\left\{z \in C:\left|z-a_{i i}\right| \leq x_{i} \sum_{j \neq i} \frac{1}{x_{j}}\left|a_{j i}\right|, i \in N\right\} .
$$




\section{Bounds for the Entries of the Inverse of a Doubly Strictly Diagonally Dominant $M$-Matrix}

In this section, upper and lower bounds for the entries of $A^{-1}$ are given when $A$ is a doubly strictly diagonally dominant $M$ matrix.

Lemma 7 (see [11, Lemma 2.2]). Let $A=\left[a_{i j}\right] \in R^{n \times n}$ be a strictly diagonally dominant $M$-matrix and let $A^{-1}=\left[\beta_{i j}\right]$. Then, for all $i \in N$,

$$
\beta_{i j} \leq \frac{\sum_{k \neq i}\left|a_{i k}\right|}{a_{i i}} \beta_{j j}, \quad j \in N, j \neq i .
$$

Next, we present a similar result for a doubly strictly diagonally dominant $M$-matrix.

Theorem 8. Let $A=\left[a_{i j}\right] \in R^{n \times n}$ be a doubly strictly diagonally dominant $M$-matrix and let $A^{-1}=\left[\beta_{i j}\right]$. Then, for all $i \in N$,

$$
\beta_{i j} \leq \widehat{h}_{i} \beta_{j j}, \quad j \in N, j \neq i
$$

Proof. If $\left(\Delta^{0} \cup \Delta^{-}\right)=\emptyset$, then $A$ is strictly diagonally dominant and the conclusion follows from Lemma 7. We next suppose that $\left(\Delta^{0} \cup \Delta^{-}\right)=\left\{i_{0}\right\}$. From Lemma 5, we get that $A X$ is a strictly diagonally dominant $M$-matrix for any $\varepsilon \in$ $\left(h_{i_{0}}, \min _{i \neq i_{0}}\left(1 / h_{i}\right)\right)$, where $X=\operatorname{diag}\left(x_{1}, x_{2}, \ldots, x_{n}\right), x_{i_{0}}=\varepsilon$, and $x_{i}=1, i \neq i_{0}$. Let $A X=\left[\bar{a}_{i j}\right]$ and $X^{-1} A^{-1}=\left[\bar{\beta}_{i j}\right]$. Then

$$
\begin{aligned}
& \bar{a}_{i j}= \begin{cases}a_{i j} \varepsilon, & j=i_{0}, i \in N, \\
a_{i j}, & j \neq i_{0}, i \in N,\end{cases} \\
& \bar{\beta}_{i j}= \begin{cases}\frac{\beta_{i j}}{\varepsilon}, & i=i_{0}, j \in N, \\
\beta_{i j}, & i \neq i_{0}, j \in N .\end{cases}
\end{aligned}
$$

If $i=i_{0}$, from Lemma 7 , we have that

$$
\frac{\beta_{i j}}{\varepsilon}=\bar{\beta}_{i j} \leq \frac{\sum_{k \neq i}\left|\bar{a}_{i k}\right|}{\bar{a}_{i i}} \bar{\beta}_{j j}=\frac{\sum_{k \neq i}\left|a_{i k}\right|}{a_{i i} \varepsilon} \beta_{j j}, \quad j \neq i ;
$$

that is,

$$
\beta_{i_{0} j} \leq \frac{\sum_{k \neq i_{0}}\left|a_{i_{0} k}\right|}{a_{i_{0} i_{0}}} \beta_{j j}=h_{i_{0}} \beta_{j j}=\widehat{h}_{i_{0}} \beta_{j j}, \quad j \neq i_{0} .
$$

If $i \neq i_{0}$ and $j=i_{0}$, from Lemma 7 , then

$$
\bar{\beta}_{i j}=\beta_{i j} \leq \frac{\sum_{k \neq i}\left|\bar{a}_{i k}\right|}{\bar{a}_{i i}} \bar{\beta}_{j j}=\frac{\left|a_{i i_{0}}\right| \varepsilon+\sum_{k \neq i, i_{0}}\left|a_{i k}\right|}{a_{i i}} \frac{\beta_{j j}}{\varepsilon},
$$

that is,

$$
\beta_{i i_{0}} \leq \frac{\left|a_{i i_{0}}\right| \varepsilon+\sum_{k \neq i, i_{0}}\left|a_{i k}\right|}{a_{i i} \varepsilon} \beta_{i_{0} i_{0}}
$$

moreover, by $\varepsilon>h_{i_{0}} \geq 1$, we have

$$
\beta_{i i_{0}} \leq \frac{\left|a_{i i_{0}}\right| \varepsilon+\sum_{k \neq i, i_{0}}\left|a_{i k}\right|}{a_{i i}} \beta_{i_{0} i_{0}} .
$$

And if $i \neq i_{0}$ and $j \neq i_{0}$, from Lemma 7, then

$$
\bar{\beta}_{i j}=\beta_{i j} \leq \frac{\sum_{k \neq i}\left|\bar{a}_{i k}\right|}{\bar{a}_{i i}} \bar{\beta}_{j j}=\frac{\left|a_{i i_{0}}\right| \varepsilon+\sum_{k \neq i, i_{0}}\left|a_{i k}\right|}{a_{i i}} \beta_{j j} ;
$$

that is,

$$
\beta_{i j} \leq \frac{\left|a_{i j}\right| \varepsilon+\sum_{k \neq i, i_{0}}\left|a_{i k}\right|}{a_{i i}} \beta_{j j}, \quad j \neq i .
$$

Hence, from inequality (30) and inequality (32) and letting $\varepsilon \rightarrow h_{i_{0}}$, we have that, for any $i \neq i_{0}$,

$$
\beta_{i j} \leq \frac{\left|a_{i i_{0}}\right| h_{i_{0}}+\sum_{k \neq i, i_{0}}\left|a_{i k}\right|}{a_{i i}} \beta_{j j}=\widehat{h}_{i} \beta_{j j}, \quad j \neq i .
$$

The conclusion follows from inequality (27) and inequality (33).

We next establish the upper and lower bounds for the diagonal entries of the inverse of a doubly strictly diagonally dominant $M$-matrix.

Theorem 9. Let $A=\left[a_{i j}\right] \in R^{n \times n}$ be a doubly strictly diagonally dominant $M$-matrix and let $A^{-1}=\left[\beta_{i j}\right]$. Then, for all $i \in N$,

$$
\frac{1}{a_{i i}} \leq \beta_{i i} \leq \frac{1}{\widehat{s}_{i}}
$$

Proof. If $\left(\Delta^{0} \cup \Delta^{-}\right)=\emptyset$, then the conclusion follows from Lemma 2.2 of [9]. We next suppose that $\left(\Delta^{0} \cup \Delta^{-}\right)=\left\{i_{0}\right\}$. Since $A$ is a doubly strictly diagonally dominant $M$-matrix, $A^{-1} \geq 0$ and $a_{i j} \leq 0, i, j \in N, i \neq j$. By $A A^{-1}=I$, we have that, for all $i \in N$,

$$
1=a_{i i} \beta_{i i}+\sum_{j \neq i} a_{i j} \beta_{j i}
$$

which implies

$$
\beta_{i i} \geq \frac{1}{a_{i i}} .
$$

Moreover, from equality (35) and Theorem 8, we have that, for any $i \neq i_{0}$,

$$
\begin{aligned}
1 & \geq a_{i i} \beta_{i i}+\sum_{j \neq i} a_{i j} \widehat{h}_{j} \beta_{i i} \\
& =\left(a_{i i}+\sum_{j \neq i} a_{i j} \widehat{h}_{j}\right) \beta_{i i} \\
& =\widehat{s}_{i} \beta_{i i} .
\end{aligned}
$$


And similar to the proof of Theorem $8, A X=\left[\bar{a}_{i j}\right]$ is a strictly diagonally dominant $M$-matrix, where $X$ is given in Lemma 5. Let $(A X)^{-1}=\left[\bar{\beta}_{i j}\right]$. Then, from $A X(A X)^{-1}=I$, we have that

$$
\begin{aligned}
1= & \bar{a}_{i_{0} i_{0}} \bar{\beta}_{i_{0} i_{0}}+\sum_{j \neq i_{0}} \bar{a}_{i_{0} j} \bar{\beta}_{j i_{0}} \\
\geq & \bar{a}_{i_{0} i_{0}} \bar{\beta}_{i_{0} i_{0}}+\sum_{j \neq i_{0}} \bar{a}_{i_{0} j} \frac{\sum_{k \neq j}\left|\bar{a}_{j k}\right|}{\bar{a}_{j j}} \bar{\beta}_{i_{0} i_{0}}(\text { by Lemma 8) } \\
= & \left(\bar{a}_{i_{0} i_{0}}+\sum_{j \neq i_{0}} \bar{a}_{i_{0} j} \frac{\sum_{k \neq j}\left|\bar{a}_{j k}\right|}{\bar{a}_{j j}}\right) \bar{\beta}_{i_{0} i_{0}} \\
= & \left(a_{i_{i} i_{0}} \varepsilon+\sum_{j \neq i_{0}} a_{i_{0} j} \frac{\left|a_{j i_{0}}\right| \varepsilon+\sum_{k \neq j, i_{0}}\left|a_{j k}\right|}{a_{j j}}\right) \frac{\beta_{i_{0} i_{0}}}{\varepsilon} \\
= & \left(a_{i_{0} i_{0}}+\frac{1}{\varepsilon} \sum_{j \neq i_{0}} a_{i_{0} j} \frac{\left|a_{j i_{0}}\right| \varepsilon+\sum_{k \neq j, i_{0}}\left|a_{j k}\right|}{a_{j j}}\right) \beta_{i_{0} i_{0}} \\
\geq & \left(a_{i_{0} i_{0}}+\frac{1}{h_{i_{0}}} \sum_{j \neq i_{0}} a_{i_{0} j} \frac{\left|a_{j i_{0}}\right| h_{i_{0}}+\sum_{k \neq j, i_{0}}\left|a_{j k}\right|}{a_{j j}}\right) \\
& \times \beta_{i_{0} i_{0}}\left(b y \varepsilon>h_{i_{0}}\right) \\
= & \left(a_{i_{0} i_{0}}+\frac{1}{\hat{h}_{i_{0}}} \sum_{j \neq i_{0}} a_{i_{0} j} \widehat{h}_{j}\right) \beta_{i_{0} i_{0}} \\
= & \widehat{s}_{i_{0}} \beta_{i_{0} i_{0}} .
\end{aligned}
$$

Hence, from inequality (37), inequality (38), and Lemma 5, we obtain that for any $i \in N$

$$
\beta_{i i} \leq \frac{1}{\widehat{s}_{i}}
$$

The conclusion follows from inequality (36) and inequality (39).

Next a lower bound of the entries of the inverse of a doubly strictly diagonally dominant $M$-matrix will be established. Firstly, a lemma is given.

Lemma 10 (see [4, Theorem 3.5]). Let $A=\left[a_{i j}\right] \in R^{n \times n}$ be $a$ weakly chained diagonally dominant $M$-matrix and let $A^{-1}=$ $\left[\beta_{i j}\right]$. Then

$$
\min _{j, k} \beta_{j k} \geq \frac{1}{a_{n n}} \prod_{i=1}^{n-1} \min \left\{l_{i}, r_{i}\right\}
$$

Theorem 11. Let $A=\left[a_{i j}\right] \in R^{n \times n}$ be a doubly strictly diagonally dominant $M$-matrix and let $A^{-1}=\left[\beta_{i j}\right]$. Then

$$
\min _{j, k} \beta_{j k} \geq \frac{1}{\omega a_{n n}} \prod_{i=1}^{n-1} \min \left\{l_{i}, \widehat{r}_{i}\right\}
$$

where

$$
\omega= \begin{cases}1, & \text { if }\left(\Delta^{-} \bigcup \Delta^{0}\right)=\emptyset, \\ \min _{i \neq i_{0}} \frac{1}{h_{i}}, & \text { if }\left(\Delta^{-} \bigcup \Delta^{0}\right)=\left\{i_{0}\right\} .\end{cases}
$$

Proof. If $\left(\Delta^{0} \cup \Delta^{-}\right)=\emptyset$, then $A$ is a strictly diagonally dominant $M$-matrix, also a weakly chained diagonally dominant $M$-matrix. The conclusion is evident from Lemma 10 . We next suppose that $\left(\Delta^{0} \cup \Delta^{-}\right)=\left\{i_{0}\right\}$. Similar to the proof of Theorem $8, A X$ is a strictly diagonally dominant $M$-matrix, where $X$ is given in Lemma 5. Let $A X=\left[\bar{a}_{i j}\right]$ and $(A X)^{-1}=$ $\left[\bar{\beta}_{i j}\right]$. By Lemma 10, we have that

$$
\min _{j, k} \bar{\beta}_{j k} \geq \frac{1}{\bar{a}_{n n}} \prod_{i=1}^{n-1} \min \left\{\frac{1}{\bar{a}_{i i}} \sum_{k=i+1}^{n}\left|\bar{a}_{k i}\right|, \frac{1}{\bar{a}_{i i}} \sum_{k=i+1}^{n}\left|\bar{a}_{i k}\right|\right\} .
$$

Moreover, note that $\min _{j, k} \beta_{j k} \geq \min _{j, k} \bar{\beta}_{j k}$ and $1 / \bar{a}_{n n} \geq$ $1 / \varepsilon a_{n n}>1 / \omega a_{n n}$; we have

$$
\min _{j, k} \beta_{j k} \geq \frac{1}{\omega a_{n n}} \prod_{i=1}^{n-1} \min \left\{\frac{1}{\bar{a}_{i i}} \sum_{k=i+1}^{n}\left|\bar{a}_{k i}\right|, \frac{1}{\bar{a}_{i i}} \sum_{k=i+1}^{n}\left|\bar{a}_{i k}\right|\right\} .
$$

And also note that, for any $i \in N$,

$$
\frac{1}{\bar{a}_{i i}} \sum_{k=i+1}^{n}\left|\bar{a}_{k i}\right|=\frac{1}{a_{i i}} \sum_{k=i+1}^{n}\left|a_{k i}\right|=l_{i} \text {. }
$$

Hence, we need only prove that $\left(1 / \bar{a}_{i i}\right) \sum_{k=i+1}^{n}\left|\bar{a}_{i k}\right| \geq \widehat{r}_{i}$ for any $i \in N$. In fact, if $i<i_{0}$, then

$$
\begin{aligned}
\frac{1}{\bar{a}_{i i}} \sum_{k=i+1}^{n}\left|\bar{a}_{i k}\right| & =\frac{1}{a_{i i}}\left(\sum_{k=i+1, k \neq i_{0}}^{n}\left|a_{i k}\right|+\left|a_{i i_{0}}\right| \varepsilon\right) \\
& \geq \frac{1}{a_{i i}}\left(\sum_{k=i+1, k \neq i_{0}}^{n}\left|a_{i k}\right|+\left|a_{i i_{0}}\right| h_{i_{0}}\right)=\widehat{r}_{i} .
\end{aligned}
$$

If $i=i_{0}$, then

$$
\frac{1}{\bar{a}_{i i}} \sum_{k=i+1}^{n}\left|\bar{a}_{i k}\right|=\frac{1}{\varepsilon a_{i i}} \sum_{k=i+1}^{n}\left|a_{i k}\right| \geq \frac{1}{\omega a_{i i}} \sum_{k=i+1}^{n}\left|a_{i k}\right|=\widehat{r}_{i} .
$$

If $i>i_{0}$, then

$$
\frac{1}{\bar{a}_{i i}} \sum_{k=i+1}^{n}\left|\bar{a}_{i k}\right|=\frac{1}{a_{i i}} \sum_{k=i+1}^{n}\left|a_{i k}\right|=r_{i}=\widehat{r}_{i} .
$$

Hence, for any $i \in N$,

$$
\frac{1}{\bar{a}_{i i}} \sum_{k=i+1}^{n}\left|\bar{a}_{i k}\right| \geq \widehat{r}_{i} .
$$

The conclusion follows from inequalities (44), (45), and (49). 


\section{Bounds for the Minimum}

\section{Eigenvalue of a Doubly Strictly Diagonally} Dominant $M$-Matrix

In this section, we give some lower bounds for $\tau(A)$ which depend only on the entries of $A$ when $A$ is a doubly strictly diagonally dominant $M$-matrix. First, for $A^{-1}=$ $\left[\beta_{i j}\right]$, we give an upper bound of $\left\|A^{-1}\right\|_{1}$, where $\left\|A^{-1}\right\|_{1}=$ $\max _{i \in N}\left\{\sum_{j=1}^{n}\left|\beta_{j i}\right|\right\}$.

Theorem 12. Let $A=\left[a_{i j}\right] \in R^{n \times n}$ be a doubly strictly diagonally dominant $M$-matrix. Then

$$
\left\|A^{-1}\right\|_{1} \leq \max _{i \in N}\left\{\frac{1}{\widehat{s}_{i}}\left(1+\sum_{j \neq i} \widehat{h}_{j}\right)\right\}
$$

Proof. Let $A^{-1}=\left[\beta_{i j}\right]$. Then

$$
\begin{aligned}
\left\|A^{-1}\right\|_{1} & =\max _{i \in N}\left\{\sum_{j} \beta_{j i}\right\} \\
& \leq \max _{i \in N}\left\{\beta_{i i}+\sum_{j \neq i} \widehat{h}_{j} \beta_{i i}\right\} \text { (by Theorem 9) } \\
& =\max _{i \in N}\left\{\left(1+\sum_{j \neq i} \widehat{h}_{j}\right) \beta_{i i}\right\} \\
& \leq \max _{i \in N}\left\{\frac{1}{\widehat{s}_{i}}\left(1+\sum_{j \neq i} \widehat{h}_{j}\right)\right\} \text { (by Theorem 10). }
\end{aligned}
$$

The proof is completed.

Theorem 13. Let $A=\left[a_{i j}\right] \in R^{n \times n}$ be a doubly strictly diagonally dominant $M$-matrix. Then

$$
\tau(A) \geq \min _{i \in n}\left\{\frac{\widehat{s}_{i}}{1+(n-1) \widehat{h}_{i}}\right\} .
$$

Proof. If $A$ is irreducible, then $A^{-1}>0$; meanwhile, from the irreducibility of $A$ and the definition of $\widehat{h}_{i}$, we have $\widehat{h}_{i}>0$ for any $i \in N$. We next consider the spectral radius $\rho\left(A^{-1}\right)$ of $A^{-1}$. From Lemma 6 , we have that there is $k_{0} \in N$ such that

$$
\left|\rho\left(A^{-1}\right)-\beta_{k_{0} k_{0}}\right| \leq \widehat{h}_{k_{0}} \sum_{k \neq k_{0}} \frac{\beta_{k k_{0}}}{\widehat{h}_{k}}
$$

which, from $\rho\left(A^{-1}\right)>\beta_{k_{0} k_{0}}[12]$, leads to

$$
\begin{aligned}
\rho\left(A^{-1}\right) & \leq \beta_{k_{0} k_{0}}+\widehat{h}_{k_{0}} \sum_{k \neq k_{0}} \frac{\beta_{k k_{0}}}{\widehat{h}_{k}} \\
& \leq \beta_{k_{0} k_{0}}+\widehat{h}_{k_{0}} \sum_{k \neq k_{0}} \beta_{k_{0} k_{0}} \text { (by Theorem 9) } \\
& =\left(1+(n-1) \widehat{h}_{k_{0}}\right) \beta_{k_{0} k_{0}} \\
& \leq \frac{1+(n-1) \widehat{h}_{k_{0}}}{\widehat{s}_{k_{0}}}(\text { by Theorem 10) } \\
& \leq \max _{i \in N}\left\{\frac{1+(n-1) h_{i}}{\widehat{s}_{i}}\right\} .
\end{aligned}
$$

Hence,

$$
\tau(A)=\frac{1}{\rho\left(A^{-1}\right)} \geq \min _{i \in n}\left\{\frac{\widehat{s}_{i}}{1+(n-1) \widehat{h}_{i}}\right\} .
$$

If $A$ is reducible, then we can obtain a doubly strictly diagonally dominant $M$-matrix $A(\epsilon)$ such that $A(\epsilon)$ is irreducible by replacing some nondiagonal zero entries of $A$ with sufficiently small negative real number $-\epsilon$. Now replace $A$ with $A(\epsilon)$ in the previous case. Let $\epsilon$ approach 0 ; the conclusion follows by the continuity of $\tau(A)$ about the entries of $A$.

From Theorems 12 and 13, we have the following result.

Theorem 14. Let $A=\left[a_{i j}\right] \in R^{n \times n}$ be a doubly strictly diagonally dominant $M$-matrix. Then

$$
\tau(A) \geq \max \{\bar{H}, \widetilde{H}\}
$$

where

$$
\begin{aligned}
& \bar{H}=\min _{i}\left\{\frac{\widehat{s}_{i}}{1+(n-1) \widehat{h}_{i}}\right\}, \\
& \widetilde{H}=\min _{i}\left\{\frac{\widehat{s}_{i}}{1+\sum_{j \neq i} \widehat{h}_{j}}\right\} .
\end{aligned}
$$

Proof. By Theorem 12 and the fact that $\rho\left(A^{-1}\right) \leq\left\|A^{-1}\right\|_{1}$, we have that

$$
\tau(A)=\frac{1}{\rho\left(A^{-1}\right)} \geq \frac{1}{\left\|A^{-1}\right\|_{1}} \geq \min _{i \in n}\left\{\frac{\widehat{s}_{i}}{1+\sum_{j \neq i} \widehat{h}_{j}}\right\}=\widetilde{H} .
$$

Hence, from Theorem 13, $\tau(A) \geq \max \{\bar{H}, \widetilde{H}\}$.

We now give upper and lower bounds for the components of the eigenvector $z$ corresponding to the minimum eigenvalue $\tau(A)$ for an irreducible doubly strictly diagonally dominant $M$-matrix. 
Theorem 15. Let $A=\left[a_{i j}\right] \in R^{n \times n}$ be an irreducible doubly strictly diagonally dominant $M$-matrix and let $A^{-1}=\left[\beta_{i j}\right]$. And let $z=\left[z_{1}, z_{2}, \ldots, z_{n}\right]^{T}$ be the positive eigenvector of $A$ corresponding to $\tau(A)$ with $\|z\|_{1}=1$. Then, for all $i \in N$,

$$
\tau(A) \min _{j, k} \beta_{j k} \leq z_{i} \leq \tau(A) \max _{j, k} \beta_{j k} .
$$

Furthermore,

$$
\max _{i, j} \frac{z_{i}}{z_{j}} \leq \max _{i, j, k} \frac{\widehat{h}_{i} \beta_{k k}}{\beta_{j k}} .
$$

Proof. It is clear that $A^{-1}$ exists and $A^{-1}>0$. From $A z=$ $\tau(A) z$ and $z>0$, we have $A^{-1} z=\rho\left(A^{-1}\right) z=\tau(A)^{-1} z$ and $z>0$; hence,

$$
z_{i}=\tau(A) \sum_{k=1}^{n} \beta_{i k} z_{k} \leq \tau(A) \max _{j, k} \beta_{j k} \sum_{k=1}^{n} z_{k}=\tau(A) \max _{j, k} \beta_{j k},
$$

where $\sum_{k=1}^{n} z_{k}=1$. The lower bound for $z_{i}$ is proved similarly. Furthermore, by Theorem 3.1 of [12],

$$
\max _{i, j} \frac{z_{i}}{z_{j}} \leq \max _{i, j, k} \frac{\beta_{i k}}{\beta_{j k}} .
$$

By Theorem $8, \beta_{i k} \leq \widehat{h}_{i} \beta_{k k}$. Hence,

$$
\max _{i, j} \frac{z_{i}}{z_{j}} \leq \max _{i, j, k} \frac{\widehat{h}_{i} \beta_{k k}}{\beta_{j k}} .
$$

The proof is completed.

\section{Example}

Consider the following system:

$$
\frac{d x}{d t}=-A x(t), \quad x(0)=x^{0}
$$

where

$$
A=\left[\begin{array}{ccccc}
1 & -0.2 & -0.2 & -0.2 & -0.6 \\
-0.2 & 1 & -0.2 & -0.2 & -0.2 \\
-0.2 & -0.2 & 1 & -0.2 & -0.2 \\
-0.2 & -0.2 & -0.2 & 1 & -0.2 \\
-0.2 & -0.2 & -0.2 & -0.2 & 1
\end{array}\right] .
$$

It is easy to verify that $A$ is an irreducible doubly strictly diagonally dominant $M$-matrix and that $\Delta^{-}=\{1\}$. Hence $A$ is not a weakly chained diagonally dominant $M$-matrix. We now establish the upper bound for the $\mathscr{L}_{1}$-norm of the solution $x(t)$. Let $A^{-1}=\left[\beta_{i j}\right]$. By Theorems 8 and 9 , we have

$$
\max _{j, k} \beta_{j k} \leq 7.5000 .
$$

By Theorem 11, we have

$$
\min _{j, k} \beta_{j k} \geq 0.0307 .
$$

By Theorem 14, we have

$$
\tau(A) \geq 0.0276 \text {. }
$$

Hence, by inequality (3) and Theorem 15, we have

$$
\mathrm{Q}=\max _{i, j} \frac{z_{i}}{z_{j}} \leq \max _{i, j, k} \frac{\widehat{h}_{i} \beta_{k k}}{\beta_{j k}} \approx 244.1406 .
$$

Hence,

$$
\|x(t)\|_{1} \leq 244.1406 e^{-0.0276 t}\left\|x^{0}\right\|_{1} .
$$

Note here that we cannot estimate the lower bound of $\tau(A)$ by using Theorem 2 (Theorem 4.1 of [4]) and Theorem 4 (Corollary 3.4 of [9]) because $A$ is not a strictly diagonally dominant $M$-matrix and not a weakly chained diagonally dominant $M$-matrix.

\section{Conflict of Interests}

The authors declare that they have no conflict of interests.

\section{Authors' Contribution}

Ming $\mathrm{Xu}$, Suhua $\mathrm{Li}$, and Chaoqian Li contributed equally to this work. All authors read and approved the final paper.

\section{Acknowledgments}

The authors are grateful to the referees for their useful and constructive suggestions. The first author is supported by Science Foundation of Guizhou Province (20132260, LKK201331, and LKK201424). The third author is supported by National Natural Science Foundations of China (11326242, 11361074), Natural Science Foundations of Yunnan Province (2013FD002), and IRTSTYN.

\section{References}

[1] A. Berman and R. J. Plemmons, Nonnegative Matrices in the Mathematical Sciences, Academic Press, New York, NY, USA, 1979.

[2] M. Fiedler and T. L. Markham, "An inequality for the Hadamard product of an $M$-matrix and an inverse $M$-matrix," Linear Algebra and Its Applications, vol. 101, pp. 1-8, 1988.

[3] R. A. Horn and C. R. Johnson, Topics in Matrix Analysis, Cambridge University Press, Cambridge, UK, 1991.

[4] P. N. Shivakumar, J. J. Williams, Q. Ye, and C. A. Marinov, "On two-sided bounds related to weakly diagonally dominant $M$ matrices with application to digital circuit dynamics," SIAM Journal on Matrix Analysis and Applications, vol. 17, no. 2, pp. 298-312, 1996.

[5] R. S. Varga, Geř́gorin and His Circles, Springer, Berlin, Germany, 2004.

[6] C. Corduneanu, Principles of Differential and Integral Equations, Chelsea, New York, NY, USA, 1988.

[7] C. Q. Li, Y. T. Li, and R. J. Zhao, "New inequalities for the minimum eigenvalue of $M$-matrices," Linear and Multilinear Algebra, vol. 61, no. 9, pp. 1267-1279, 2013. 
[8] W. Walter, Differential and Integral Inequalities, Springer, New York, NY, USA, 1970.

[9] G. X. Tian and T. Z. Huang, "Inequalities for the minimum eigenvalue of $M$-matrices," Electronic Journal of Linear Algebra, vol. 20, pp. 291-302, 2010.

[10] R. S. Varga, "Minimal Gerschgorin sets," Pacific Journal of Mathematics, vol. 15, pp. 719-729, 1965.

[11] Y. Li, F. Chen, and D. Wang, "New lower bounds on eigenvalue of the Hadamard product of an $M$-matrix and its inverse," Linear Algebra and its Applications, vol. 430, no. 4, pp. 1423-1431, 2009.

[12] H. Minc, Nonnegative Matrices, John Wiley \& Sons, New York, NY, USA, 1987. 


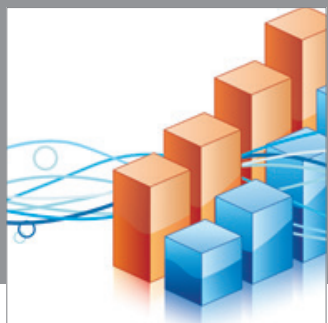

Advances in

Operations Research

mansans

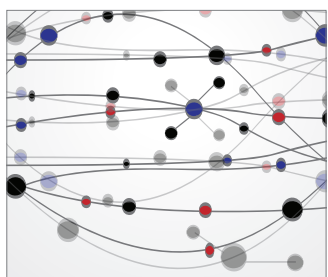

The Scientific World Journal
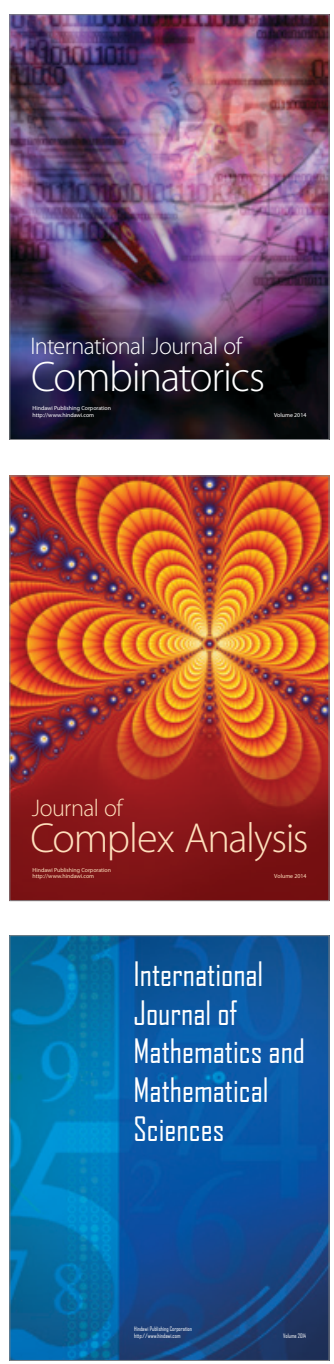
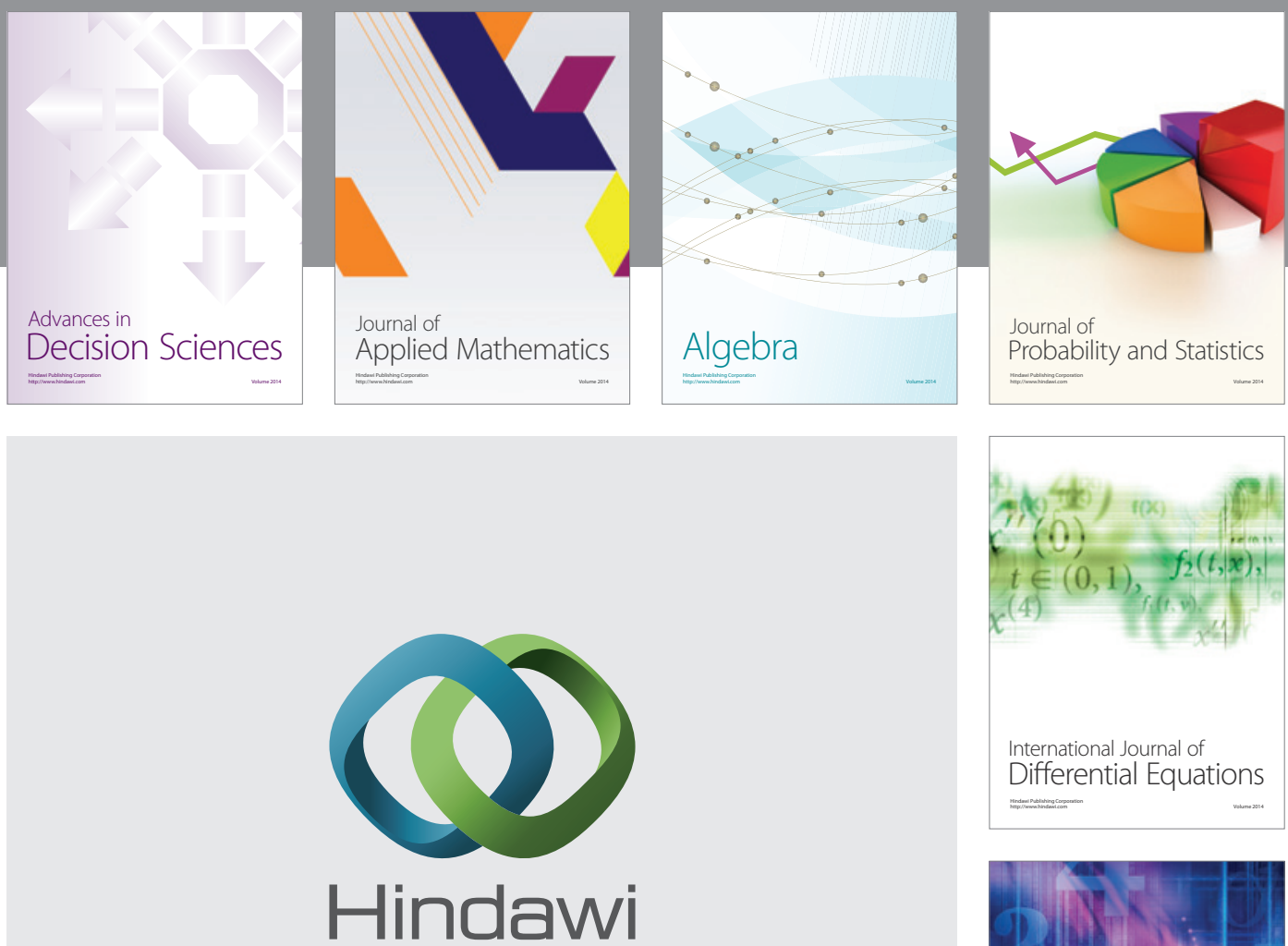

Submit your manuscripts at http://www.hindawi.com
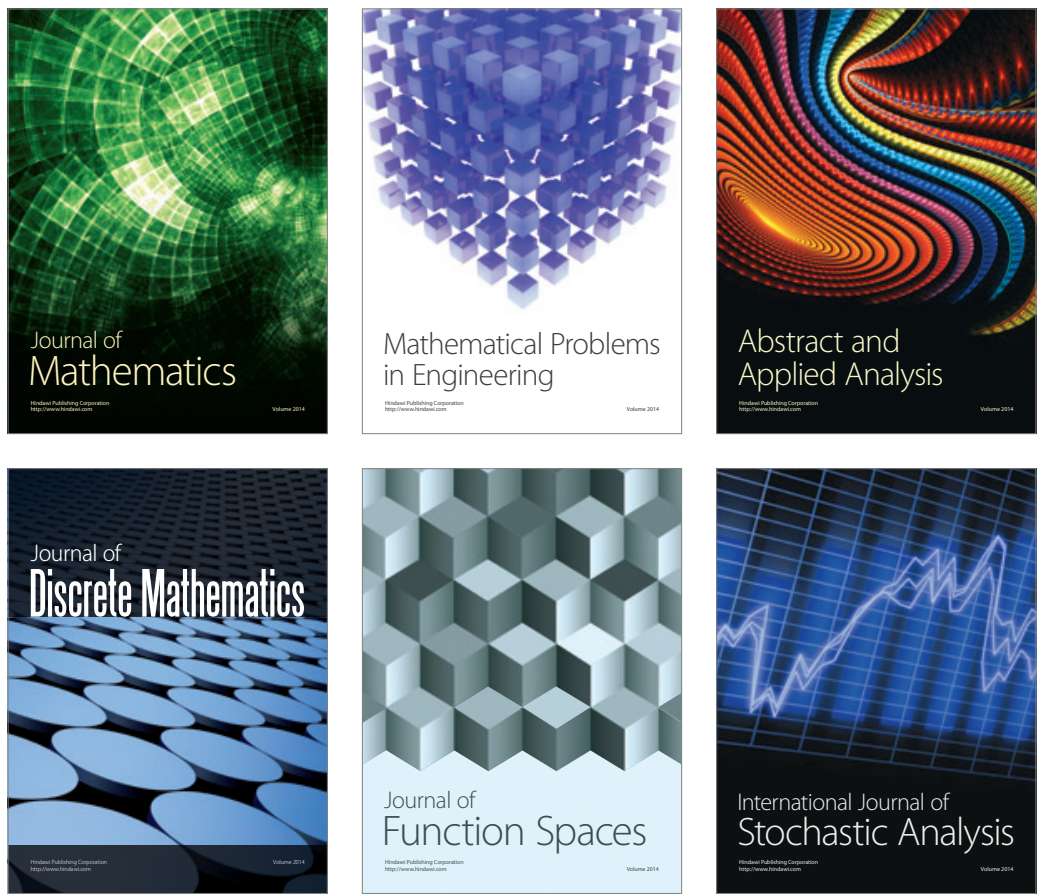

Journal of

Function Spaces

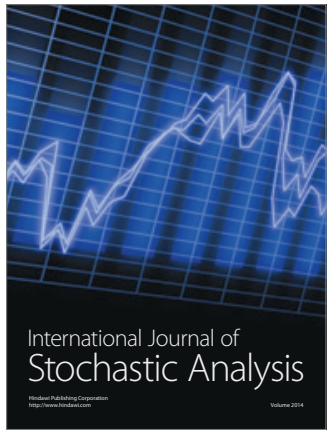

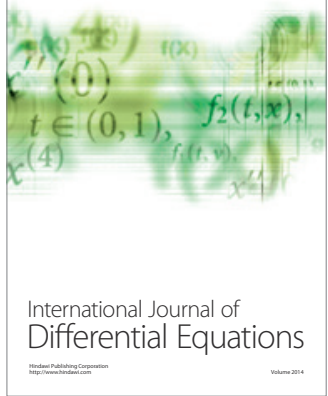
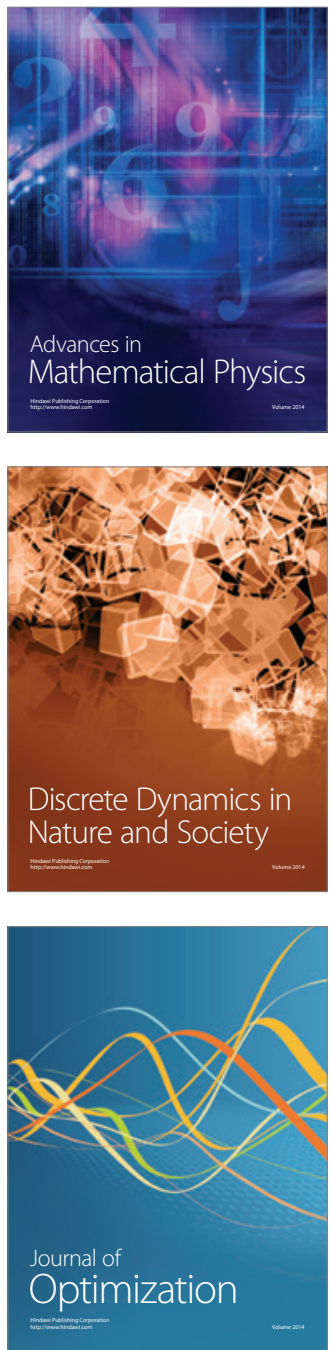